\title{
Downregulated METTL14 Expression Correlates with Breast Cancer Tumor Grade and Molecular Classification
}

\author{
Xiao-Fang Dong, ${ }^{1}$ Yan Wang, ${ }^{1}$ Bi-Fei Huang, ${ }^{2}$ Gui-Nv Hu, ${ }^{3}$ Jun-Kang Shao, ${ }^{2}$ Qian Wang, \\ Chih-Hsin Tang $\left(1,{ }^{4,5,6}\right.$ and Chao-Qun Wang ${ }^{2}$ \\ ${ }^{1}$ Department of Medical Oncology, Affiliated Dongyang Hospital of Wenzhou Medical University, Dongyang, Zhejiang, China \\ ${ }^{2}$ Department of Pathology, Affiliated Dongyang Hospital of Wenzhou Medical University, Dongyang, Zhejiang, China \\ ${ }^{3}$ Department of Surgical Oncology, Affiliated Dongyang Hospital of Wenzhou Medical University, Dongyang, Zhejiang, China \\ ${ }^{4}$ Graduate Institute of Basic Medical Science, China Medical University, Taichung, Taiwan \\ ${ }^{5}$ Department of Pharmacology, School of Medicine, China Medical University, Taichung, Taiwan \\ ${ }^{6}$ Department of Biotechnology, College of Health Science, Asia University, Taichung, Taiwan
}

Correspondence should be addressed to Chih-Hsin Tang; chtang@mail.cmu.edu.tw and Chao-Qun Wang; chaoqunw869@163.com

Xiao-Fang Dong and Yan Wang contributed equally to this work.

Received 29 August 2020; Revised 6 October 2020; Accepted 10 October 2020; Published 20 October 2020

Academic Editor: Hua Tang

Copyright (c) 2020 Xiao-Fang Dong et al. This is an open access article distributed under the Creative Commons Attribution License, which permits unrestricted use, distribution, and reproduction in any medium, provided the original work is properly cited.

\begin{abstract}
It is unclear whether the methyltransferase-like 14 (METTL14) protein promotes or suppresses cancer growth. We examined the association between METTL14 expression, cancer progression, and patient prognosis in a total of 398 breast cancer tissue specimens. Significantly fewer cancer tissue specimens compared with normal breast tissue expressed high levels of METTL14 (52.8\% vs. $75.0 \%)$. METTL14 expression was negatively associated with tumor grade and positively associated with patient age, estrogen, and progesterone receptor status. High METTL14 expression was more common in luminal A and luminal B tissue $(75.9 \%$ and $60.8 \%$, respectively), compared with human epidermal growth factor receptor 2- (HER2-) enriched and triple-negative breast cancer (TNBC) samples $(38.2 \%$ and $18.6 \%$, respectively). In multiple logistic regression analysis, independent predictors of METTL14 expression in breast cancer included higher tumor grade (odds ratio $($ OR) $=0.494$, 95\% confidence interval $(\mathrm{CI}): 0.289-0.844 ; P=0.010)$, TNBC subtype $(\mathrm{OR}=0.109,95 \% \mathrm{CI}: 0.054-0.222 ; P<0.001)$, and HER2-enriched subtype $(\mathrm{OR}=0.298,95 \% \mathrm{CI}: 0.156-0.567 ; P<0.001)$. No clear relationship was observed between patient prognosis and METTL14 expression. It appears that downregulated METTL14 expression in breast cancer is associated with tumor grade and molecular classification.
\end{abstract}

\section{Introduction}

Breast cancer is the leading cause of cancer-related death among women [1]. Breast cancer is a biologically diverse disease, reflected by different molecular subtypes that have different biological behaviors and clinical treatment options [2-4]. The different subtypes include luminal A and luminal $\mathrm{B}$, human epidermal growth factor receptor 2- (HER2-) enriched, and triple-negative or basal-like; immunohistochemistry (IHC) can assess standard biomarkers (estrogen receptor (ER), progesterone receptor (PR), and HER2) as well as Ki-67 status (a marker of cell proliferation) [2, 5]. Triple-negative breast cancer (TNBC) has a more aggressive clinical course and poorer prognosis than other types of breast cancer, and currently there is no effective treatment other than chemotherapy [6-9]. Thus, studying the molecules related to the occurrence and progression of breast cancer and their clinical significance is of great significance for guiding the treatment of breast cancer, especially TNBC. 
The catalytic protein methyltransferase-like 14 (METTL14) participates in mediate cellular $N^{6}$-methyladenosine $\left(\mathrm{m}^{6} \mathrm{~A}\right)$ deposition [10] and is closely related to the occurrence and development of malignant tumors [11-23]. METTL14 plays a complex role in malignant tumors: low levels of METTL14 expression are found in hepatocellular carcinoma [13], colorectal cancer [14, 16, 17], bladder cancer [15], gastric cancer [18], childhood ETV6/RUNX1-positive acute lymphoblastic leukemia [19], and papillary thyroid carcinoma [20], where METTL14 functions as a tumor suppressor [13-18], whereas levels of METTL14 expression are high in pancreatic cancer $[21,22]$ and acute myeloid leukemia [23], where this protein functions as a tumor promoter. In breast cancer, contrasting findings have been reported from analyses of the expression and function of METTL14. In one study, significantly decreased METTL14 expression was observed in patients with breast cancer compared with healthy controls, and overexpression of METTL14 inhibited breast cancer cell viability, colony formation, and migration [24]. Conversely, in another study, significantly upregulated METTL14 expression was observed in breast cancer tissue compared with normal tissue, and METTL14 overexpression enhanced the migration and invasion capacities of breast cancer cells [25], while other researchers have reported significantly upregulated levels of METTL14 and $\mathrm{m}^{6} \mathrm{~A}$ in breast cancer cells and tissues, and the modulation of METTL14 by long noncoding RNA LINC00942 (LNC942) promoted breast cancer initiation and progression [26]. Thus, further research is needed to better understand the expression and function of METTL14 in breast cancer. This study performed an IHC analysis of METTL14 expression in breast cancer tissue samples obtained from 398 Chinese Han women, to clarify the expression of METTL14 in breast cancer and its clinicopathological and prognostic significance.

\section{Materials and Methods}

2.1. Patients and Tissue Samples. Breast cancer tissue samples were obtained from 398 untreated Chinese Han women aged 24-90 years (median 50 years) who underwent surgery in the Affiliated Dongyang Hospital of Wenzhou Medical University (Dongyang, Zhejiang, China) between 2007 and 2019. Twenty-four samples of adjacent normal breast tissue were also obtained following surgical resection. A pathohistological diagnosis was made according to the breast tumor classification criteria of the World Health Organization [27]. Histological grading was based on the Scarff-BloomRichardson system [28]. According to ER, PR, HER2, and $\mathrm{Ki}-67$ status, the samples were classified into 4 subtypes [2, 5]: luminal A $\left(\mathrm{ER}^{+} / \mathrm{PR}^{+}[\geq 20 \%] / \mathrm{HER} 2^{-}, \mathrm{Ki} 67<14 \%\right)$; luminal $\mathrm{B}$, containing hormone receptor-positive cases that did not meet the conditions of luminal A; HER2-enriched (ER-, $\mathrm{PR}^{-}$, and $\left.\mathrm{HER}_{2}^{+}\right)$; or TNBC $\left(\mathrm{ER}^{-}, \mathrm{PR}^{-}\right.$, and $\mathrm{HER} 2^{-}$). Follow-up information was available for 228 breast cancer patients with a median follow-up time of 60 months (range, 6-70 months). The Medical Ethics Committee of the Affiliated Dongyang Hospital of Wenzhou Medical University approved this study. All study methods satisfied the relevant guidelines and regulations issued by the Affiliated Dongyang Hospital of Wenzhou Medical University.

2.2. Tissue Array Preparation. We followed the methods described by Wang et al. [29]. In brief, the Quick-Ray ${ }^{\circledR}$ UT06 (Unitma Co., Ltd., Seoul, Korea) tissue microarray system and the Quick-Ray premade recipient block (UB-06) wax model were used to prepare tissue specimens ( $1 \mathrm{~mm}$ in diameter). Two representative sites from each breast cancer tissue sample were selected for sampling.

2.3. IHC Analysis. IHC staining of paraffin-embedded tissue sections used the Envision System (Dako, Glostrup, Denmark), as described previously [5, 30]. Primary antibodies consisted of anti-METTL14 mouse monoclonal antibody (clone CL4252, diluted at 1:1000; Abcam, Cambridge, England), ready-to-use anti-ER rabbit monoclonal antibody (clone SP1, Dako), ready-to-use anti-PR mouse monoclonal antibody (clone PgR636, Dako), HercepTest (Dako), and ready-to-use anti-Ki-67 mouse monoclonal antibody (clone MIB-1, Dako).

2.4. Assessment of Staining. Each entire section was scanned and scored independently by 2 pathologists. The intensity of nuclear staining for METTL14 was assessed in breast tissue. Staining intensity was scored on a 4-point scale from 0 (negative) to 1 (weak), 2 (moderate), or 3 (strong). High METTL14 expression was defined as a staining intensity of positive invasive cancer cells of 2 or 3 [31]. A case was considered to be ER- or PR-positive if the percentage of positive invasive cancer cells (nuclear staining) was $\geq 1 \%$ [32]. HER2 status was determined by the 2018 American Society of Clinical Oncology/College of American Pathologists guidelines for HER2 testing in breast cancer [33].

2.5. Patient Follow-Up. We followed the methods of Wang et al. [29]. In brief, each patient was followed up postoperatively by telephone call and thereafter at 6 monthly hospital appointments; follow-up was discontinued in the event of the patient's death. A diagnosis of local breast cancer recurrence was made by clinical or histology results. Relapse-free survival (RFS) was defined as the time from surgery to relapse/metastasis; overall survival (OS) was the time from surgery to death (excluding non-tumor-related deaths).

2.6. Flow Diagram. A flowchart of the study methodology is shown in Figure 1.

2.7. Statistical Analysis. Statistical analyses were conducted using SPSS software version 19.0 (SPSS Inc., Chicago, IL, USA). Between-group differences were compared using Pearson's chi-square test for qualitative variables. The relative risk was expressed by odds ratios (ORs) and their 95\% confidence intervals (CIs). The independent correlation factor of METTL14 expression in breast cancer was assessed by multiple logistic regression analysis. RFS and OS rates were estimated by the Kaplan-Meier method and compared using log-rank testing. $P<0.05$ was considered to be statistically significant. 


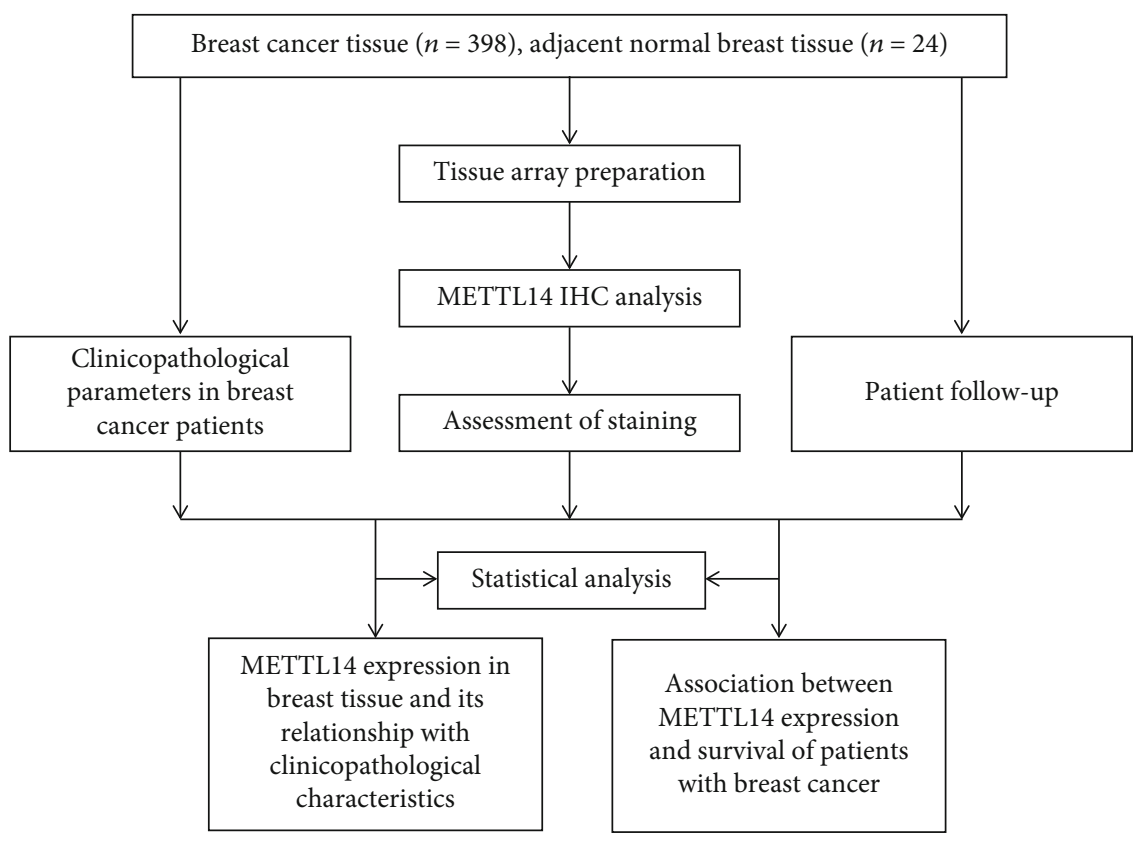

FIgURE 1: Flow diagram of the study methodology.

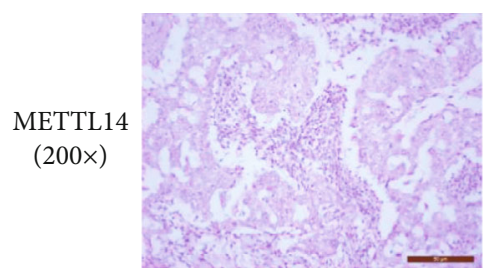

No expression (0)

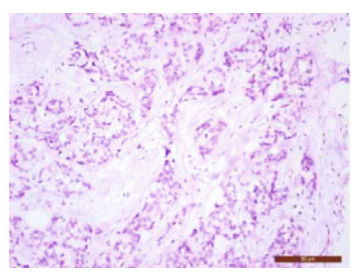

Weak expression $(1+)$

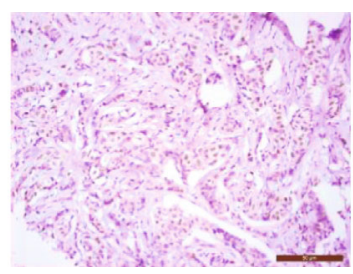

Moderate expression (2+)

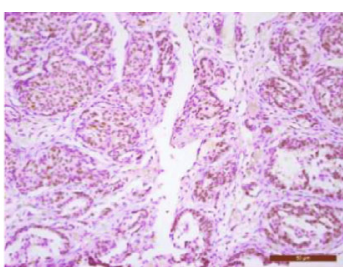

Strong expression $(3+)$

FIGURE 2: METTL14 expression levels in breast cancer patients. Breast cancer tissue specimens were immune-stained with anti-METTL14 antibody, photographed using an optical microscope, and scored from 0 (negative) to 3 (strong) for nuclear staining intensity of METTL14 expression.

\section{Results}

3.1. METTL14 Expression in Breast Tissue and Its Relationship with Clinicopathological Characteristics. Around half of the breast cancer tissue specimens $(52.8 \%$, 210/398) expressed high METTL14 expression, compared with $75.0 \%(18 / 24)$ of normal breast tissue specimens (Figure 2); the between-group difference was significant $(P=0.034)$ (Table 1$)$.

As shown in Table 2, high METTL14 expression was identified in $64.2 \%(174 / 271)$ of grade I-II tumor tissue and in $28.3 \%(36 / 127)$ of grade III tumor tissue; the betweengroup difference was highly significant $(P<0.001)$. In younger-aged patients ( $\leq 35$ years) and in those whose tumors were ER- or PR-negative, rates of high METTL14 expression were $31.8 \%$ (7/22), 28.6\% (48/168), and $39.2 \%$ $(82 / 209)$, respectively, which were significantly lower than the corresponding rates among older-aged patients $(>35$ years; 54.0\% [203/376]), ER-positive tissue (70.4\%, $162 / 230)$, and PR-positive tissue $(67.7 \%, 128 / 189)$ $(P=0.043, P<0.001$, and $P<0.001$, respectively). Among
TABLE 1: METTL14 expression in breast tissue specimens.

\begin{tabular}{lccc}
\hline Group & No. & \multicolumn{2}{c}{ METTL14 expression } \\
& & $(\%)$ & $\begin{array}{c}\text { Low } \\
\text { High expression }\end{array}{ }^{\dagger}, n$ \\
\hline $\begin{array}{l}\text { Normal tissue } \\
\text { Cancerous }\end{array}$ & 24 & $6(25.0 \%)$ & $18(75.0 \%)$ \\
tissue & 398 & $188(47.2 \%)$ & $210(52.8 \%)^{*}$ \\
\hline
\end{tabular}

${ }^{\dagger}$ High METTL14 expression was defined as a nuclear staining intensity of positive invasive cancer cells of 2 or 3 [17]. ${ }^{*} P<0.05$ vs. normal breast tissue.

the 4 molecular subtypes, TNBC was associated with the lowest rate of high METTL14 expression (18.6\%, 18/97), compared with rates of $43.3 \%$ (29/67) for HER2-enriched tumors, 60.8\% (59/97) for luminal B, and 75.9\% (104/137) for luminal A tumors $(P<0.001)$. In multiple logistic regression analysis, independent predictors of METTL14 expression in breast cancer included higher tumor grade (odds ratio $(\mathrm{OR})=0.494, \quad 95 \%$ confidence interval $(\mathrm{CI})$ : $0.289-0.844 ; P=0.010)$, the presence of the TNBC subtype $(\mathrm{OR}=0.109,95 \% \mathrm{CI}: 0.054-0.222 ; \quad P<0.001)$, and the 
TABLE 2: Association of METTL14 expression with clinicopathological parameters in breast cancer patients.

\begin{tabular}{|c|c|c|c|}
\hline Parameters & $\begin{array}{l}\text { No. of } \\
\text { patients }\end{array}$ & $\begin{array}{l}\text { High METTL14 } \\
\text { expression, } n(\%)\end{array}$ & $\begin{array}{c}p \\
\text { value }\end{array}$ \\
\hline \multicolumn{4}{|l|}{ Age (years) } \\
\hline$\leq 35$ & 22 & $7(31.8 \%)$ & \multirow{2}{*}{0.043} \\
\hline$>35$ & 376 & $203(54.0 \%)$ & \\
\hline \multicolumn{4}{|c|}{ Tumor size $(\mathrm{cm})$} \\
\hline$\leq 2$ & 176 & $99(56.3 \%)$ & \multirow{3}{*}{0.365} \\
\hline $2-5$ & 201 & $102(50.7 \%)$ & \\
\hline$>5$ & 21 & $9(42.9 \%)$ & \\
\hline \multicolumn{4}{|c|}{ Lymph node metastases } \\
\hline No & 203 & $99(48.8 \%)$ & \multirow{2}{*}{0.103} \\
\hline Yes & 195 & $111(56.9 \%)$ & \\
\hline \multicolumn{4}{|l|}{ Tumor grade } \\
\hline I-II & 271 & $174(64.2 \%)$ & \multirow{2}{*}{$<0.001$} \\
\hline III & 127 & $36(28.3 \%)$ & \\
\hline \multicolumn{4}{|l|}{ Tumor stage } \\
\hline I & 103 & $59(57.3 \%)$ & \multirow{4}{*}{0.552} \\
\hline II & 201 & $102(50.7 \%)$ & \\
\hline III & 94 & $49(52.1 \%)$ & \\
\hline IV & 0 & $0(0.00)$ & \\
\hline \multicolumn{4}{|c|}{ Estrogen receptor } \\
\hline Negative & 168 & $48(28.6 \%)$ & \multirow{2}{*}{$<0.001$} \\
\hline Positive & 230 & $162(70.4 \%)$ & \\
\hline \multicolumn{4}{|c|}{ Progesterone receptor } \\
\hline Negative & 209 & $82(39.2 \%)$ & \multirow{2}{*}{$<0.001$} \\
\hline Positive & 189 & $128(67.7 \%)$ & \\
\hline \multicolumn{4}{|c|}{ HER2 expression } \\
\hline $\begin{array}{l}\text { Negative } \\
\left(0-1^{+}\right)\end{array}$ & 191 & $93(48.7 \%)$ & \multirow{3}{*}{0.095} \\
\hline $\begin{array}{l}\text { Equivocal } \\
\left(2^{+}\right)\end{array}$ & 109 & $67(61.5 \%)$ & \\
\hline $\begin{array}{l}\text { Positive } \\
\left(3^{+}\right)\end{array}$ & 98 & $50(51.0 \%)$ & \\
\hline \multicolumn{4}{|c|}{ Molecular classification } \\
\hline Luminal A & 137 & $104(75.9 \%)$ & \multirow{4}{*}{$<0.001$} \\
\hline Luminal B & 97 & $59(60.8 \%)$ & \\
\hline $\begin{array}{l}\text { HER2- } \\
\text { enriched }\end{array}$ & 67 & $29(43.3 \%)$ & \\
\hline TNBC & 97 & $18(18.6 \%)$ & \\
\hline
\end{tabular}

${ }^{*}$ High METTL14 expression was defined as a nuclear staining intensity of positive invasive cancer cells of 2 or 3 [17]. Abbreviations: HER2: human epidermal growth factor receptor 2; TNBC: triple-negative breast cancer.

presence of the HER2-enriched subtype ( $\mathrm{OR}=0.298,95 \%$ CI: $0.156-0.567 ; P<0.001)$.

3.2. No Association between METTL14 Expression and Survival of Patients with Breast Cancer. When we examined the data for potential associations between levels of METTL14 expression and survival rates in a cohort of 228 breast cancer patients, 5-year RFS and OS rates were 79.8\% and $88.6 \%$, respectively. As shown in Figure 3, no clear asso- ciations were observed between METTL14 expression and survival. The 137 patients with high levels of METTL14 expression had a mean RFS of 54.0 months and an estimated 5 -year RFS rate of 79.6\%; corresponding values in the 91 patients whose tumors expressed low levels of METTL14 were 53.2 months and $80.2 \%$, respectively $(P=0.955$; Figure 3(a)). Mean OS was 57.9 months (with an estimated 5 -year OS rate of $89.8 \%$ ) in the patients with high levels of METTL14 expression and 57.0 months (with an estimated 5 -year OS rate of $86.8 \%$ ) in those with low levels of METTL14 $(P=0.478$; Figure 3(b)).

\section{Discussion}

Several studies have reported that METTL14 plays a complex role in the occurrence and development of malignant tumors [11-23]. For example, in hepatocellular carcinoma, RFS is adversely affected by downregulated levels of METTL14 expression [13], while a reduction in $\mathrm{m}^{6} \mathrm{~A}$ mRNA methylation caused by the METTL14 loss-of-function R298P mutation increases the proliferation and tumorigenicity of endometrial cancer cells [34]. In contrast, METTL14 is highly expressed in acute myeloid leukemia cells and promotes leukemogenesis via $\mathrm{m}^{6} \mathrm{~A}$ modification [23]. Findings from three investigations into METTL14 expression and function in breast cancer are controversial, with one study showing that METTL14 acts as a tumor suppressor [24], while the other two show that METTL14 promotes cancer growth $[25,26]$.

Our findings revealed that METTL14 expression was significantly downregulated in women with higher-grade tumors. Since such tumors are known to be more aggressive and to have a poor prognosis [28], this finding indicates that the downregulation of METTL14 expression may be closely related to highly invasive breast cancer and a poor prognosis. Our results also revealed significantly decreased levels of METTL14 expression in ER- and PR-negative disease, which was closely and negatively correlated with hormone receptor expression. Furthermore, evidence has shown that overexpression of METTL14 in the ER- and PR-negative breast cancer cell line MDA-MB-231 inhibits its proliferation and migration ability [24]. Since hormone receptor-negative breast cancer patients are unsuitable candidates for endocrine therapy, further research should examine whether these patients may benefit from the exogenous overexpression of METTL14. We also found in this study that METTL14 expression was significantly decreased in younger-aged women ( $\leq 35$ years), who have been shown to have more aggressive breast cancer and a worse prognosis [35, 36]. Our analysis showed that having higher-grade disease, the TNBC subtype or the HER2-enriched subtype is independently correlated with low METTL14 expression in breast cancer. These results suggest that METTL14 expression in breast cancer is correlated with tumor grade and molecular classification, although our survival analysis failed to reveal any association between METTL14 expression and survival. More research is needed to determine how METTL14 expression affects the prognosis of breast cancer patients. 


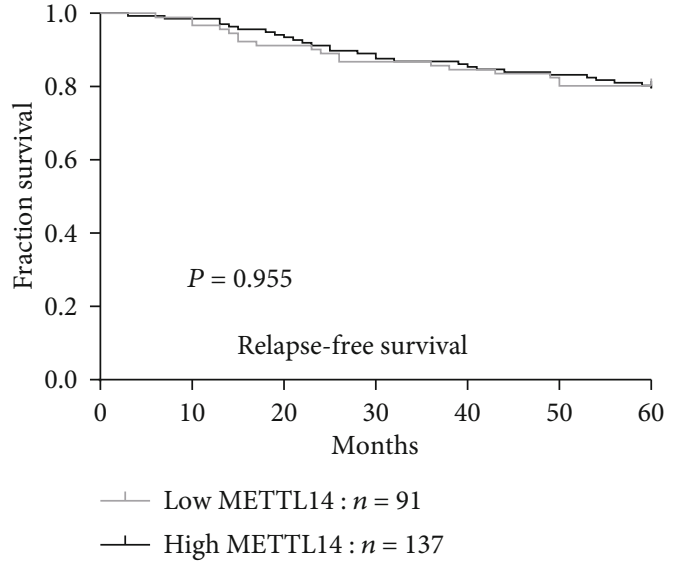

(a)

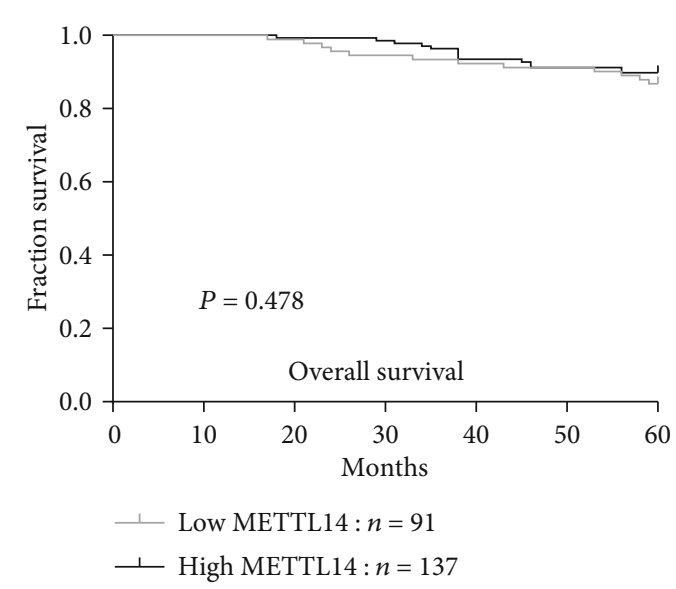

(b)

FIGURE 3: METTL14 expression is not associated with the survival of patients with breast cancer. The associations of METTL14 expression with relapse-free survival (a) and overall survival (b).

TNBC disease is hormone receptor-negative and HER2negative, is associated with a younger age at diagnosis, a higher tumor grade, a more aggressive clinical course, and poorer prognosis than other types of breast cancer, and is not amenable to endocrine therapy or HER2-targeted agents, such as trastuzumab [6-9]. All of these factors highlight the need for new treatment options for TNBC. Our findings showing that METTL14 expression was the lowest in TNBC compared with other subtypes of breast cancer suggests that it may be worth targeting this protein in the treatment of patients with TNBC.

\section{Abbreviations}

$\begin{array}{ll}\text { METTL14: } & \text { Methyltransferase-like } 14 \\ \text { ER: } & \text { Estrogen receptor } \\ \text { PR: } & \text { Progesterone receptor } \\ \text { HER2: } & \text { Human epidermal growth factor receptor } 2 \\ \text { RFS: } & \text { Relapse-free survival } \\ \text { OS: } & \text { Overall survival } \\ \text { IHC: } & \text { Immunohistochemistry } \\ \text { TNBC: } & \text { Triple-negative breast cancer. }\end{array}$

\section{Data Availability}

All data generated or analyzed during this study are included in this published article.

\section{Conflicts of Interest}

The authors declare no conflicts of interest.

\section{Authors' Contributions}

CQW and XFD designed the experiments. YW and QW performed the experiments. $\mathrm{CQW}$ and $\mathrm{BFH}$ analyzed the data. XFD, GNH, and JKS were responsible for management of the clinical samples and clinicopathological information of patients. CQW wrote the manuscript. CHT revised the manuscript. All authors have read and approved the final manuscript. Xiao-Fang Dong and Yan Wang contributed equally to this work.

\section{Acknowledgments}

This work was supported by grants from the National Natural Science Foundation of China (No. 81802660) and the Zhejiang Public Welfare Technology Application Social Development Project (No. LGF19H160003). We would like to thank Iona J. MacDonald from China Medical University for her editing of this manuscript.

\section{References}

[1] F. Bray, J. Ferlay, I. Soerjomataram, R. L. Siegel, L. A. Torre, and A. Jemal, "Global cancer statistics 2018: GLOBOCAN estimates of incidence and mortality worldwide for 36 cancers in 185 countries," CA: a Cancer Journal for Clinicians, vol. 68, no. 6, pp. 394-424, 2018.

[2] S. Park, J. S. Koo, M. S. Kim et al., "Characteristics and outcomes according to molecular subtypes of breast cancer as classified by a panel of four biomarkers using immunohistochemistry," Breast, vol. 21, no. 1, pp. 50-57, 2012.

[3] A. Prat, E. Pineda, B. Adamo et al., "Clinical implications of the intrinsic molecular subtypes of breast cancer," Breast, vol. 24, Suppl 2, pp. S26-S35, 2015.

[4] A. G. Waks and E. P. Winer, "Breast cancer treatment: a review," JAMA, vol. 321, no. 3, pp. 288-300, 2019.

[5] C. Q. Wang, C. H. Tang, H. T. Chang et al., "Fascin-1 as a novel diagnostic marker of triple-negative breast cancer," Cancer Medicine, vol. 5, no. 8, pp. 1983-1988, 2016.

[6] K. R. Bauer, M. Brown, R. D. Cress, C. A. Parise, and V. Caggiano, "Descriptive analysis of estrogen receptor (ER)negative, progesterone receptor (PR)-negative, and HER2negative invasive breast cancer, the so-called triple-negative phenotype," Cancer, vol. 109, no. 9, pp. 1721-1728, 2007.

[7] P. Boyle, "Triple-negative breast cancer: epidemiological considerations and recommendations," Annals of Oncology, vol. 23, Supplement 6, pp. vi7-vi12, 2012. 
[8] S. K. Pal, B. H. Childs, and M. Pegram, "Triple negative breast cancer: unmet medical needs," Breast Cancer Research and Treatment, vol. 125, no. 3, pp. 627-636, 2011.

[9] C. Y. Li, P. Wang, S. Zhang, Y. Liu, and J. Zhang, "Clinicopathological features and prognosis of triple-negative breast cancer," Zhonghua zhong liu za zhi [Chinese Journal of Oncology], vol. 35, no. 6, pp. 463-467, 2013.

[10] J. Liu, Y. Yue, D. Han et al., “A METTL3-METTL14 complex mediates mammalian nuclear RNA N6-adenosine methylation," Nature Chemical Biology, vol. 10, no. 2, pp. 93-95, 2014.

[11] H. Shi, J. Wei, and C. He, "Where, when, and how: contextdependent functions of RNA methylation writers, readers, and erasers," Molecular Cell, vol. 74, no. 4, pp. 640-650, 2019.

[12] Q. Lan, P. Y. Liu, J. Haase, J. L. Bell, S. Huttelmaier, and T. Liu, "The critical role of RNA m6A methylation in cancer," Cancer Research, vol. 79, no. 7, pp. 1285-1292, 2019.

[13] J. Z. Ma, F. Yang, C. C. Zhou et al., "METTL14 suppresses the metastatic potential of hepatocellular carcinoma by modulating $\mathrm{N}(6)$-methyladenosine-dependent primary microRNA processing," Hepatology, vol. 65, pp. 529-543, 2016.

[14] X. Chen, M. Xu, X. Xu et al., "METTL14 suppresses CRC progression via regulating N6-methyladenosine-dependent primary miR-375 processing," Molecular Therapy : the journal of the American Society of Gene Therapy, vol. 28, no. 2, pp. 599-612, 2020.

[15] C. Gu, Z. Wang, N. Zhou et al., "Mettl14 inhibits bladder TIC self-renewal and bladder tumorigenesis through N6methyladenosine of Notch1," Molecular Cancer, vol. 18, no. 1, p. 168, 2019.

[16] X. Yang, S. Zhang, C. He et al., "METTL14 suppresses proliferation and metastasis of colorectal cancer by down-regulating oncogenic long non-coding RNA XIST," Molecular Cancer, vol. 19, no. 1, p. 46, 2020.

[17] X. Chen, M. Xu, X. Xu et al., "METTL14-mediated N6methyladenosine modification of SOX4 mRNA inhibits tumor metastasis in colorectal cancer," Molecular Cancer, vol. 19, no. 1, p. 106, 2020.

[18] C. Zhang, M. Zhang, S. Ge et al., "Reduced m6A modification predicts malignant phenotypes and augmented Wnt/PI3KAkt signaling in gastric cancer," Cancer Medicine, vol. 8, no. 10, pp. 4766-4781, 2019.

[19] C. Sun, L. Chang, C. Liu, X. Chen, and X. Zhu, “The study of METTL3 and METTL14 expressions in childhood ETV6/RUNX1-positive acute lymphoblastic leukemia," Molecular Genetics \& Genomic Medicine, vol. 7, article e00933, 2019.

[20] J. Hou, H. Shan, Y. Zhang, Y. Fan, and B. Wu, “m(6)A RNA methylation regulators have prognostic value in papillary thyroid carcinoma," American Journal of Otolaryngology, vol. 41, no. 4, p. $102547,2020$.

[21] M. Wang, J. Liu, Y. Zhao et al., "Upregulation of METTL14 mediates the elevation of PERP mRNA N6 adenosine methylation promoting the growth and metastasis of pancreatic cancer," Molecular Cancer, vol. 19, no. 1, p. 130, 2020.

[22] F. Kong, X. Liu, Y. Zhou et al., "Downregulation of METTL14 increases apoptosis and autophagy induced by cisplatin in pancreatic cancer cells," The International Journal of Biochemistry \& Cell Biology, vol. 122, p. 105731, 2020.

[23] H. Weng, H. Huang, H. Wu et al., "METTL14 inhibits hematopoietic stem/progenitor differentiation and promotes leukemogenesis via mRNA m6A modification," Cell Stem Cell, vol. 22, no. 2, pp. 191-205.e9, 2018, e9.
[24] L. Wu, D. Wu, J. Ning, W. Liu, and D. Zhang, "Changes of N6methyladenosine modulators promote breast cancer progression," BMC Cancer, vol. 19, no. 1, p. 326, 2019.

[25] D. Yi, R. Wang, X. Shi, L. Xu, Y. Yilihamu, and J. Sang, "METTL14 promotes the migration and invasion of breast cancer cells by modulating N6-methyladenosine and hsa-miR-146a-5p expression," Oncology Reports, vol. 43, no. 5, pp. 1375-1386, 2020.

[26] T. Sun, Z. Wu, X. Wang et al., "LNC942 promoting METTL14-mediated m6A methylation in breast cancer cell proliferation and progression," Oncogene, vol. 39, no. 31, pp. 5358-5372, 2020.

[27] S. R. E. I. Lakhani, S. J. Schnitt, P. H. Tan, and M. J. van de Vijver, WHO Classification of Tumours of the Breast, IARC Press, Lyon, Fourth edition, 2012.

[28] C. W. Elston and I. O. Ellis, "Pathological prognostic factors in breast cancer. I. The value of histological grade in breast cancer: experience from a large study with long-term follow-up," Histopathology, vol. 19, no. 5, pp. 403-410, 1991.

[29] C. Q. Wang, Y. Wang, B. F. Huang et al., "High expression of both resistin and fascin-1 predicts a poor prognosis in patients with colorectal cancer," BioMed Research International, vol. 2020, Article ID 8753175, 7 pages, 2020.

[30] C. Q. Wang, Y. Li, B. F. Huang et al., "EGFR conjunct FSCN1 as a novel therapeutic strategy in triple-negative breast cancer," Scientific Reports, vol. 7, no. 1, p. 15654, 2017.

[31] C. Q. Wang, B. F. Huang, Y. Wang, G. R. Hu, Q. Wang, and J. K. Shao, "Expression of HMGB1 protein in breast cancer and its clinicopathological significance," Chinese Journal of Pathology, vol. 49, no. 1, pp. 57-61, 2020.

[32] T. H. Nguyen, V. H. Nguyen, T. L. Nguyen, C. Qiuyin, and T. H. Phung, "Evaluations of biomarker status changes between primary and recurrent tumor tissue samples in breast cancer patients," BioMed Research International, vol. 2019, Article ID 7391237, 7 pages, 2019.

[33] A. C. Wolff, M. E. H. Hammond, K. H. Allison et al., "Human epidermal growth factor receptor 2 testing in breast cancer: American Society of Clinical Oncology/College of American Pathologists Clinical Practice Guideline Focused Update," Journal of Clinical Oncology : official journal of the American Society of Clinical Oncology, vol. 36, no. 20, pp. 2105-2122, 2018.

[34] J. Liu, M. A. Eckert, B. T. Harada et al., "m6A mRNA methylation regulates AKT activity to promote the proliferation and tumorigenicity of endometrial cancer," Nature Cell Biology, vol. 20, no. 9, pp. 1074-1083, 2018.

[35] H. A. Azim Jr. and A. H. Partridge, "Biology of breast cancer in young women," Breast Cancer Research, vol. 16, no. 4, p. 427, 2014.

[36] Z. Anastasiadi, G. D. Lianos, E. Ignatiadou, H. V. Harissis, and M. Mitsis, "Breast cancer in young women: an overview," Updates in Surgery, vol. 69, no. 3, pp. 313-317, 2017. 